\title{
The pupillary light reflex in normal subjects
}

\author{
C. J. K. ELLIS
}

From St Thomas's Hospital, London SE1

SUMMARY In 19 normal subjects the pupillary reflex to light was studied over a range of stimulus intensities by infrared electronic pupillography and analysed by a computer technique. Increasing stimulus intensity was associated with an increase in direct light reflex amplitude and maximum rate of constriction and redilatation. Latency from stimulus to onset of response decreased with increasing stimulus intensity. The normal range for each of these parameters is given and the significance of these results in clinical pupillary assessment discussed.

The technique of infrared pupillometry ${ }^{1}$ has allowed the normal pupillary response to light to be studied in detail. Lowenstein and Friedman ${ }^{2}$ have shown that in response to light the pupil constricts after a latent period and that the length of this latent period, the amplitude of the response, and the speed of the pupillary constriction are dependent on the stimulus intensity employed. These findings have subsequently been confirmed. ${ }^{3-5}$

Borgmann ${ }^{6}$ gave $95 \%$ confidence limits in defining the effect of age on reflex amplitude and constriction and redilatation velocity. There has been no study in which a statistical analysis of the normal pupillary light reflex over a range of stimulus intensities has enabled the definition of the normal light reaction.

The introduction of computer analysis of averaged pupillary reflexes has allowed a more accurate estimation of selected parameters of the pupillary reaction to light. This technique has been used in a study of anisocoria in normal subjects. ${ }^{7}$ This study reports the methodology and results from a series of 19 normal subjects studied over a range of stimulus intensities by infrared pupillometry and defines a normal range in these subjects.

\section{Materials and methods}

CONTROL SUBJECTS

Nineteen healthy subjects were investigated by infrared pupillometry. They were aged 18 to 48 years, and 12 were female. Their corrected visual acuity was normal in every case. In one case there was anisometropia of uncorrected vision.

Correspondence to Dr. C. J. K. Ellis, King's College Hospital, Denmark Hill, London SE5.

\section{PUPILLOMETRY}

A Whittaker Series 1800 binocular infrared television pupillometer was used in this study. All recordings were made in darkness with no correction for refractive error. The eyes were illuminated from a lowintensity, invisible infrared source and observed by means of a closed circuit television system sensitive to infrared light. The pupils were displayed on television monitor screens providing instantaneous feedback of the quality of the pupil images. The pupillometer system incorporated circuitry that recognised the maximum vertical pupillary diameter and placed a white crescent on one edge of the pupil. The correct positioning of this crescent confirmed that measurements of pupillary diameter would be accurate and without interference from eyelids or iris. The maximum vertical pupillary diameter of both eyes was displayed on a dual strip chart recorder and also recorded on magnetic tape for computer analysis. The maximum vertical pupillary diameter was measured by the pupillometer system by counts of the number of television lines intersected by the recognised pupil. This system incorporated 625 lines, and the estimated precision in measurements of pupil diameter was approximately $0.03 \mathrm{~mm}$. The pupillometer imaged the pupil every $20 \mathrm{~ms}$.

The pupillometer was calibrated by recording 2 model pupils the diameters of which were measured with a travelling microscope to be $2.20 \mathrm{~mm}$ and $7.90 \mathrm{~mm}$. These 2 diameters were chosen as they lay at either end of the range of pupil diameter commonly encountered. The 2 measured diameters were given to the computer. In addition 2 standard voltage calibrations approximately equivalent to the 2 model pupil diameters were recorded on the data tape. As 
(a)

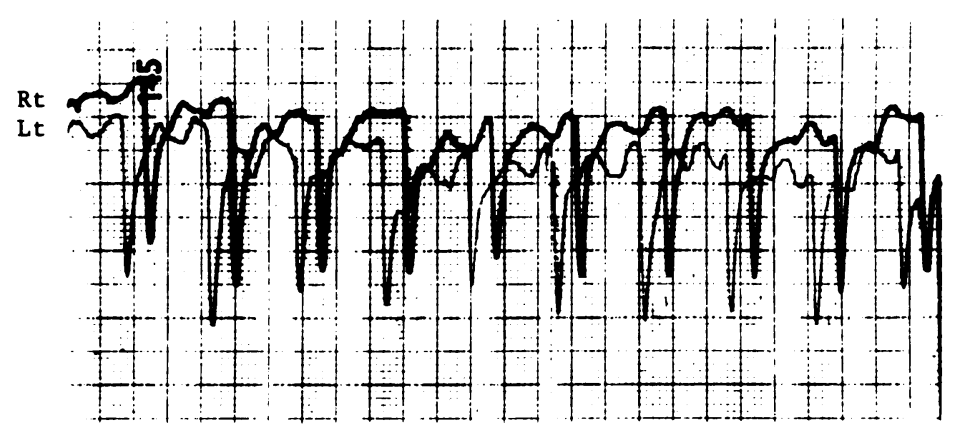

(b)

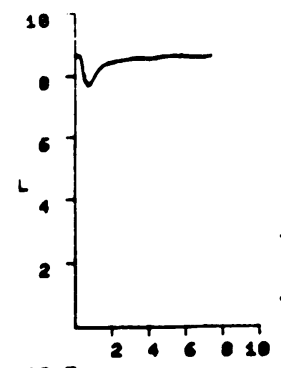

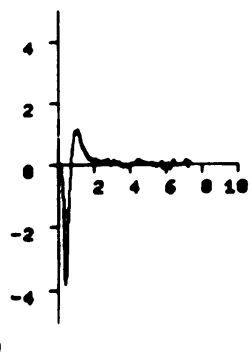

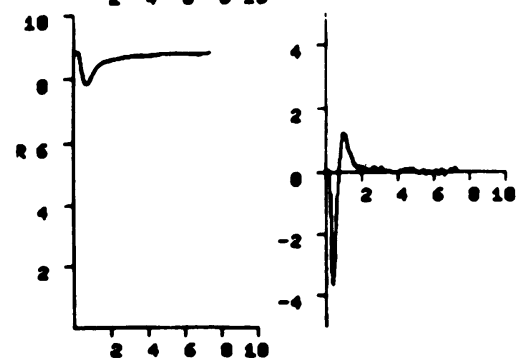

Fig. 1 (a) Train of 10 pupillary responses elicited by stimulation of the right eye. Downward deflections indicate decrease in pupillary diameter. Stimuli are delivered every 8 seconds. (b) Example of computer analysis of the averaged pupillary response for (a).

For explanation see text. part of the calibration programme the computer gave numerical values for these standard voltage calibrations. These values could be compared on every occasion recordings were made. This allowed detection of fluctuations in tape speed or pupillometer gain at any recording session throughout the period of the study. In addition the electrical calibrations were repeated during the recording session before each run of pupillary reflexes was elicited. In this way the calibration was repeated every time the tape recorder was started.

The output from the pupillometer was recorded on a 4-channel Yasec CD 110 cassette data tape recorder with separate channels for each eye, stimulus events, and a reference channel to compensate for variations in tape speed during replay.

All subjects were dark-adapted for $\mathbf{3 0}$ minutes in red goggles before recording. A comfortable position was adopted with the head supported on a chin rest and the eyes fixating a distant, dim red light by means of a mirror system. The surroundings were quiet, at a constant temperature, and the technique was fully explained to the subject before recording.

A $100 \mathrm{~ms}$ white light stimulus converging to a $2.0 \mathrm{~mm}$ beam at the midpupillary point, was delivered $7^{\circ} 31^{\prime}$ temporal to the visual axis every 8 seconds, thus impinging on the nasal retina. Two Sylvania tungsten concentrated arc lamps were used as the light source giving an intensity of approximately $\mathbf{5 5 0}$ candles $/ \mathrm{m}^{2}$ at the pupil. The stimulus length was adjusted on the pupillometer photostimulator which controlled the 2 Uniblitz electronic shutters, movements of which gave the stimulus almost square-wave characteristics. Movements of the shutter were also recorded on the stimulus channel of the data tape. The intensity of the 2 stimulus lamps was regularly 


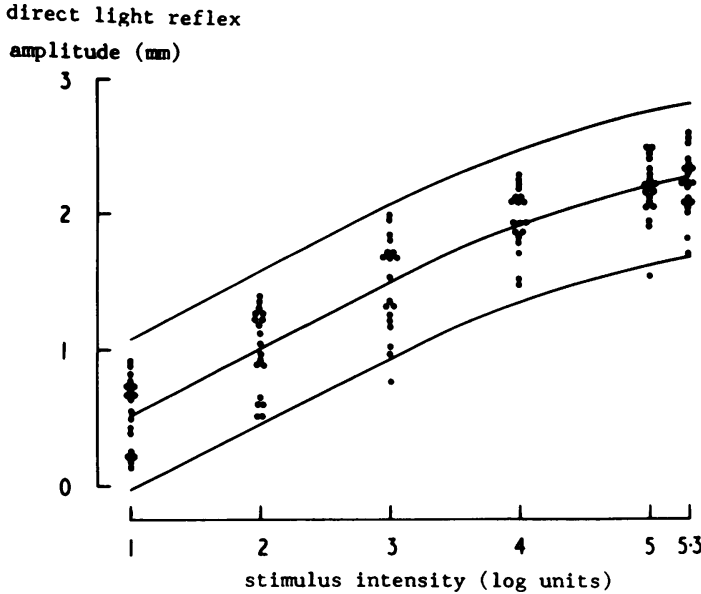

Fig. 2 The relationship between direct light reflex amplitude and stimulus intensity $\left(Y=-0.12+0.66 X-0.04 X^{2}\right)$. The lines indicate the mean and $95 \%$ confidence limits. The black dots are the values from the left eye of the 19 subjects.

measured with a photometer to ensure equal stimulus intensity in the 2 eyes. Six light intensities were employed, variation in stimulus intensity being achieved by filtering the 2 stimulus lamps with neutral density filters. Between 6 and 10 stimuli were delivered at each level of light intensity.

A pause between increments of stimulus intensity allowed dark adaptation to be maintained. Care was taken to ensure that the nonstimulated eye was kept in darkness. If stray light spread to this eye, recordings were repeated with the nonstimulated eye patched.

\section{COMPUTER ANALYSIS}

The data tape was replayed into a Varian V72 digital computer with compensation for variations in tape speed by utilising a reference input channel. Before processing, the analog output of the pupillometer was converted to digital form. The computer was programmed to average the pupillary responses at each level of stimulus intensity and to give numerical values for several parameters of this averaged response. An example of the computer print-out is shown in Fig. 1. In (a) the paper record of a train of 10 pupillary reflexes elicited by stimulation of the right eye is shown. In (b) the computer analysis of the average of these responses is shown. The uppermost 2 graphs refer to the left eye and represent the averaged response. In the first graph pupil diameter in $\mathrm{mm}$ is plotted against time in seconds. The change in diameter occurring during a light reflex is shown. The stimulus is delivered at 0 seconds. From this plot derive the values for resting diameter, reflex

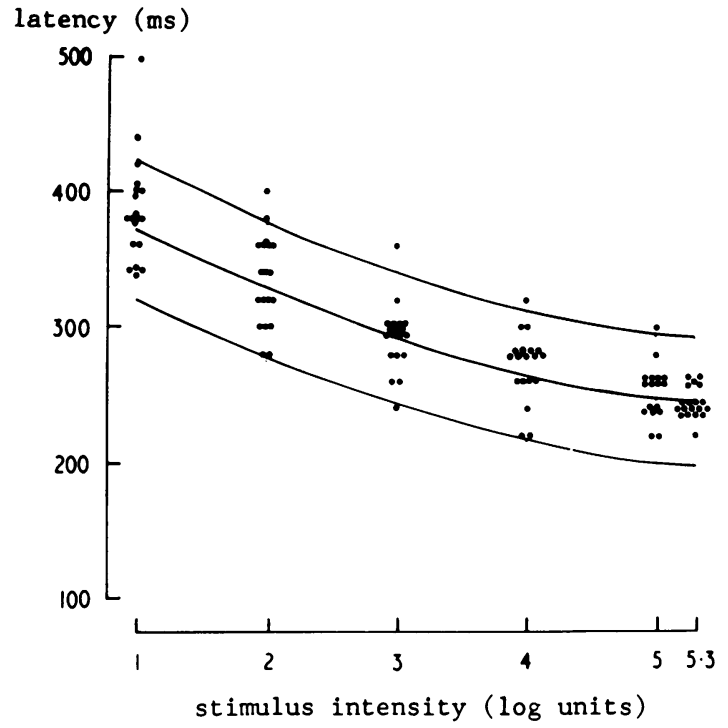

Fig. 3 The relationship between latency and stimulus intensity $\left(Y=445 \cdot 7-22 \cdot 9 X+76 \cdot 2 X^{2}\right)$. The lines indicate the mean and $95 \%$ confidence limits. The black dots are the values from the left eye of the 19 subjects.

amplitude, and latency from stimulus to onset of response. The second graph shows rate of change of pupillary diameter in $\mathrm{mm} / \mathrm{s}$ against time in seconds. From this plot derive the maximum rates of constriction and dilatation.

\section{STATISTICAL ANALYSIS}

The relationships between the measured parameters of the light reflex were expressed mathematically by linear regression analysis or second order polynomials as appropriate, by means of standard techniques. The normal range was defined as the mean $\pm 2 \mathrm{SD}$.

\section{Results}

In all subjects the averaged pupillary response was of similar shape (Fig. 1b), showing that pupillary constriction occurred after a latent period after stimulation. After constriction, pupillary redilatation occurred, and the prestimulus resting diameter was reached. The rate of pupillary constriction increased rapidly to a maximum and then decreased progressively until the redilatation phase ensued. Similarly redilatation velocity increased to a maximum and then progressively decreased as the resting diameter was approached.

\section{LIGHT REFLEX AMPLITUDE}

The amplitude of the direct light reflex increased with increasing stimulus intensity (Fig. 2). Assessment of 


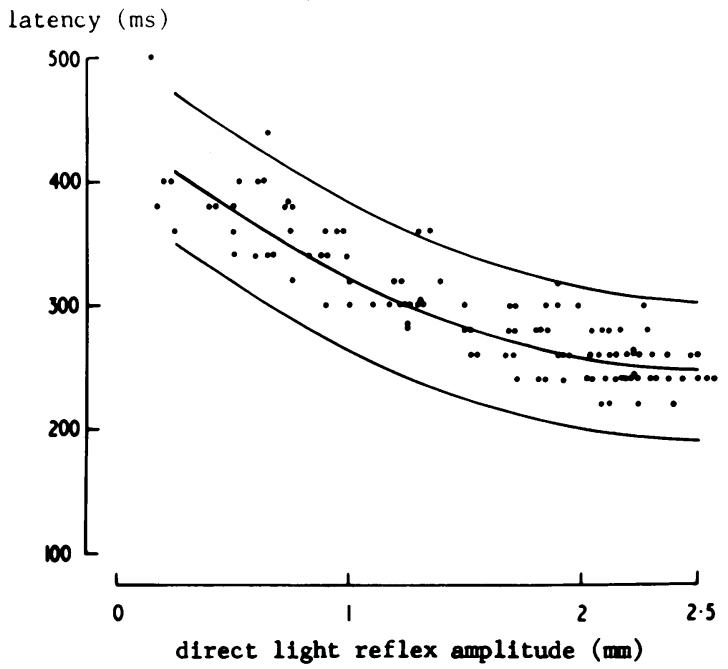

Fig. 4 The relationship between latency and direct light reflex amplitude $\left(Y=477-150 \cdot 3 X+27 \cdot 4 X^{2}\right)$. The lines indicate the mean and $95 \%$ confidence limits. The black dots are the values from the left eye of the 19 subjects.

the data indicated that, although a linear relationship between these variables was statistically significant, second order polynomial analysis yielded a line of better fit with a tendency towards a maximum reflex amplitude at the higher stimulus intensities. This maximum could be identified reliably in only a small number of the control subjects as individuals.

The $95 \%$ confidence limits define the normal range of reflex amplitude. All subjects achieved a measurable pupillary reflex at the lowest level of stimulus intensity. However, at this level the amplitude of the individual response in the series elicited was variable. By contrast the variation between the individual reflex amplitudes in the series elicited at the higher stimulus intensities was consistently small. The value for amplitude of the consensual response was generally smaller than the direct; detailed results have been published elsewhere.?

\section{LATENCY}

Latency from stimulus to onset of response was found to decrease with increasing stimulus intensity (Fig. 3). Statistical analysis similar to that applied to reflex amplitude showed that a second order polynomial provided the line of best fit. This showed that at the higher stimulus intensities latency tended to reach a minimum value. As with reflex amplitude it was not always possible to identify a minimum latency with confidence.

Latency was also found to decrease with increasing light reflex amplitude (Fig. 4). This relationship was

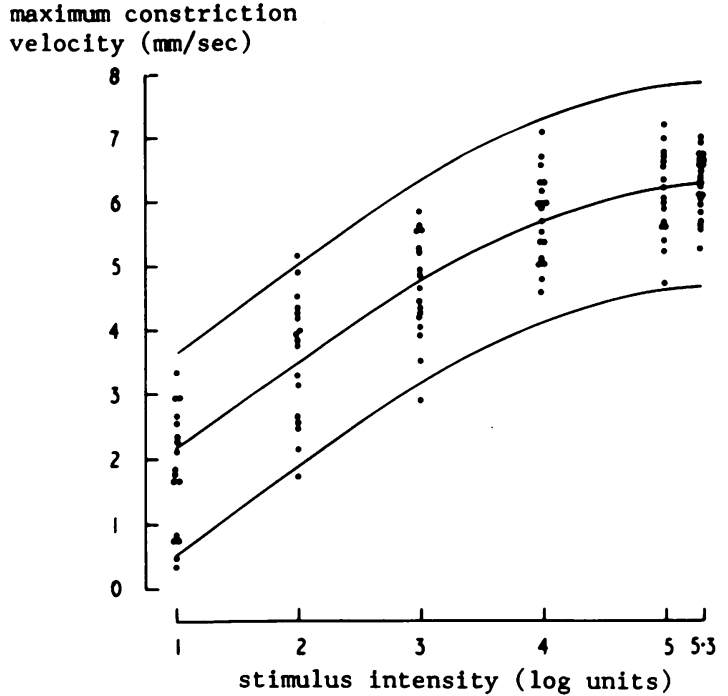

Fig. 5 The relationship between maximum constriction velocity and stimulus intensity $\left(Y=0 \cdot 15+2 \cdot 0 X-0 \cdot 17 X^{2}\right)$. The lines indicate the mean and $95 \%$ confidence limits. The black dots are the values from the left eye of the 19 subjects.

similar to that against stimulus intensity with latency tending toward a minimum value at higher reflex amplitude.

A wide range of latencies was encountered in this study. Values of $500 \mathrm{~ms}$ and $220 \mathrm{~ms}$ were recorded at the extremes of this range. In the control subjects a mean decrease in latency of $140 \mathrm{~ms}$ (range 240 to $100 \mathrm{~ms}$ ) was found over the range of stimulus intensities employed. The latency of the consensual response was not significantly different from that of the direct.

\section{MAXIMUM RATE OF PUPILLARY CONSTRICTION}

The maximum rate of pupillary constriction (maximum constriction velocity) increased with increasing stimulus intensity (Fig. 5). The line of best fit was given by a second order polynomial, and this indicated that at the higher levels of stimulus intensity the maximum constriction velocity tended toward a maximum value.

The maximum rate of pupillary constriction also increased with increasing light reflex amplitude, again tending to reach a maximum value at the higher reflex amplitudes (Fig. 6).

MAXIMUM RATE OF PUPILLARY REDILATATION The maximum rate of pupillary redilatation (maximum redilatation velocity) increased with increasing stimulus intensity (Fig. 7). Again a second order polynomial gave the line of best fit to the points and showed that at the higher stimulus intensities re- 


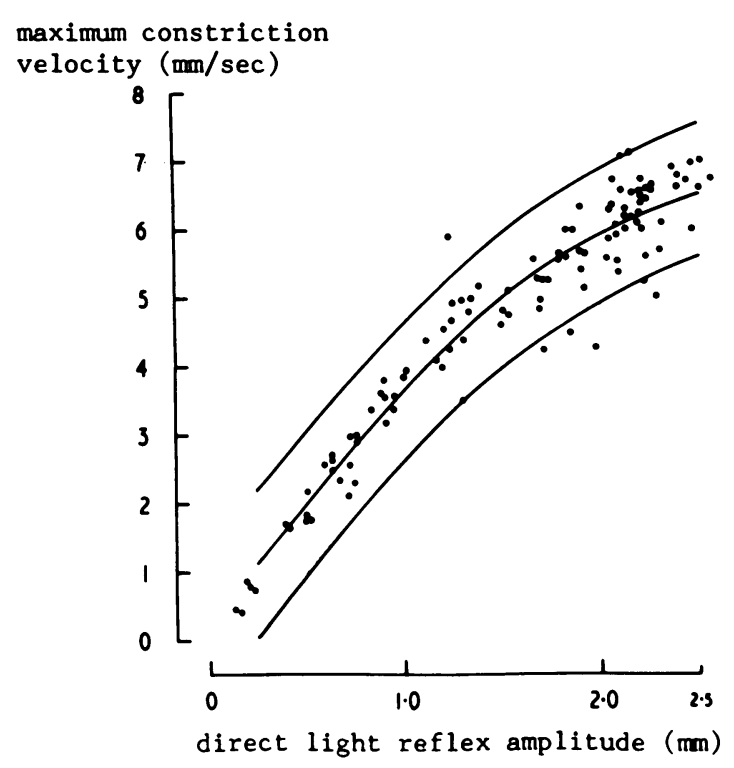

Fig. 6 The relationship between maximum constriction velocity and direct light reflex amplitude $(Y=0 \cdot 08+4 \cdot 28 X$ $\left.-0.69 X^{2}\right)$. The lines indicate the mean and $95 \%$ confidence limits. The black dots are the values from the left eye of the 19 subjects.

dilatation velocity tended to reach a maximum value.

Maximum redilatation velocity showed a similar relationship with light reflex amplitude. At the higher reflex amplitudes the maximum redilatation velocity tended toward a maximum value. (Fig. 8).

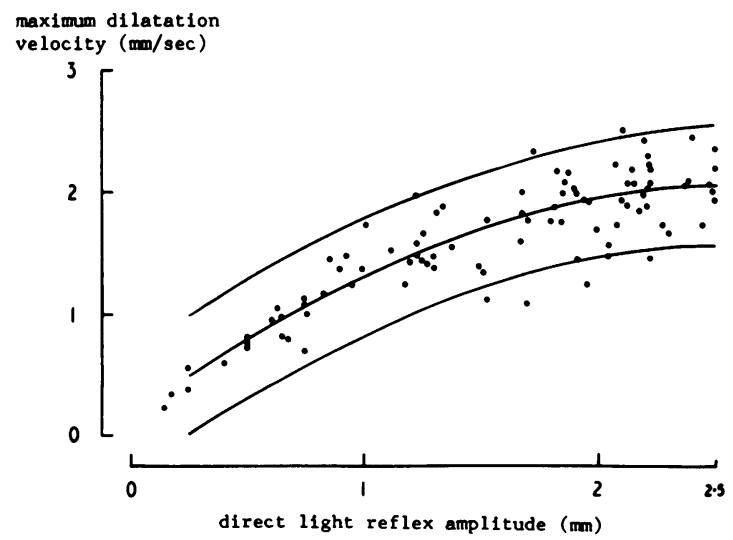

Fig. 8 The relationship between maximum redilatation velocity direct light reflex amplitude $(Y=0 \cdot 14+1 \cdot 42 X$ $\left.-0 \cdot 26 X^{2}\right)$. The lines indicate the mean and $95 \%$ confidence limits. The black dots are the values from the left eye of the 19 subjects.

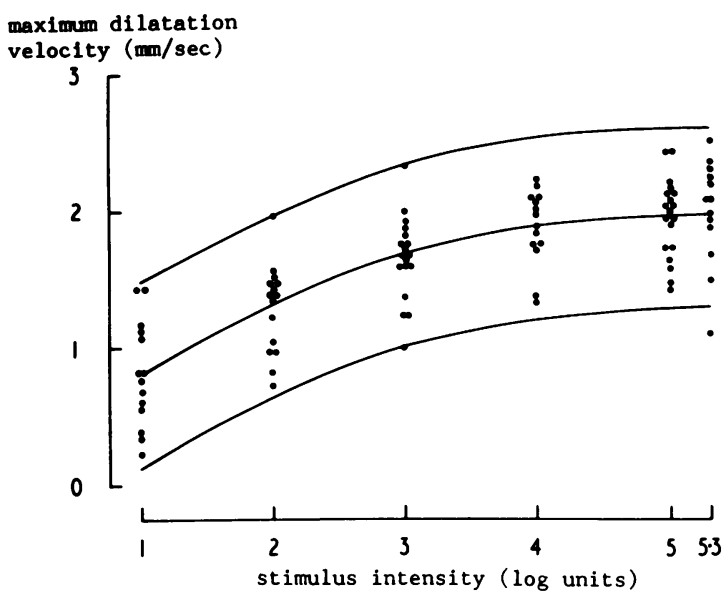

Fig. 7 The relationship between maximum redilatation velocity and stimulus intensity $\left(Y=0 \cdot 16+0 \cdot 72 X-0 \cdot 07 X^{2}\right)$ The lines indicate the mean and $95 \%$ confidence limits. The black dots are the values from the left eye of the 19 subjects.

\section{Discussion}

This study has confirmed the findings of Lowenstein and Loewenfeld' showing that with increasing stimulus intensity there is an increase in direct light reflex amplitude, a decrease in latency from stimulus to onset of response, and an increase in the maximum rate of pupillary constriction. This study has shown that redilatation velocity also increases with increasing stimulus intensity.

The subjects were examined in the dark-adapted condition with controlled stimulus intensity. The results, analysed to give a statistical normal range of the mean \pm 2 SD at each intensity level, indicate that the definition of the normal pupillary response to light must be related to stimulus intensity. In clinical testing a significant bilateral pupillary abnormality may be undetectable because of the variability both of the normal light reflex and of clinical testing conditions. In uniocular abnormalities, however, comparisons of the reactions from the 2 eyes to the same stimulus intensity may show a subtle pupillary abnormality undetectable on assessment of the affected eye alone. Previous studies ${ }^{8}$ have shown that the swinging light test ${ }^{9}$ yields reliable information regarding uniocular defects in the afferent pupillary pathway.

This study has shown that latency and constriction and redilatation velocity have a similar relationship to stimulus intensity and to direct light reflex amplitude. For a reflex of any given amplitude the latency and constriction and redilatation velocity fall within a 
defined range. Consequently for a light reflex of low amplitude a slow constriction velocity and a long latency is normal. Thus the common clinical observation of a sluggish pupillary reaction to light indicates abnormality only if associated with a normal light reflex amplitude or elicited by an inappropriate stimulus intensity. In studies on the afferent pupillary defect $^{8}$ no abnormal light reflex was recorded in which there was a significant dissociation between light reflex amplitude, latency and pupillary constriction, and redilatation velocity-all being affected equally. In this situation, therefore, abnormality will be seen as a light reflex the characteristics of which are inappropriate to the stimulus intensity employed. There have been no similar studies on the effects of lesions of the efferent pupillary pathways.

The pupillary latency is long; the shortest recorded in this study was $220 \mathrm{~ms}$. Most of the latency is due to slow iris muscle constriction. In the pigeon, with a striated muscle iris sphincter, the minimum latency recorded is around $60 \mathrm{~ms} .^{10}$ That study also showed that direct electrical stimulation of the ciliary ganglion of the cat, with a smooth muscle iris sphincter, resulted in a latency of around 180 to $200 \mathrm{~ms}$. These findings imply that the majority of the latency in normal subjects is due to delay in iris smooth muscle contraction and that a relatively small part is due to conduction along the pupillary reflex pathway.
I thank Professor S. E. Smith, Dr Shirley A. Smith. Dr R. W. Ross Russell, and Professor W. I. McDonald for their advice throughout this study. I also thank Mr P. Bourne, who devised the computer programme, and Miss $\mathrm{J}$. Lace for secretarial assistance.

This work formed part of a thesis accepted by the University of London for the degree of MD in 1980.

\section{References}

1 Lowenstein O. Loewenfeld I. Electronic pupillography. Arch Ophthalmol 1958; 59: 352-63.

2 Lowenstein O. Friedman ED. Pupillographic studies. Arch Ophthalmol 1942; 27: 969-93.

3 Lowenstein O. Loewenfeld I. In; Davson H, ed. The Eye. New York: Academic Press, 1969: chapter 9: 255-337.

4 Alpern M. McCready DW, Barr L. The dependance of the photopupil response on Flash duration and intensity. J Gen Physiol 1963; 47: 265-78.

5 Feinberg R. Podolak E. In: Behaviour, Aging and the Nervous System. Welford AT. Birren JE, eds. Springfield: Thomas, 1965.

6 Borgmann H. Abhangigkeit der Parameter der Liditriaktion vom Lebensalter. Albrecht von Graefes Arch Klin Ophthalmol 1972; 185: $1-10$.

7 Smith SA. Ellis CJK. Smith SE. Inequality of the direct and consensual light reflexes in normal subjects. Br J Ophthalmol 1979; 7: 523-7.

8 Ellis CJK. The afferent pupillary defect in acute optic neuritis. $J$ Neurol Neurosurg Psychiatry 1979; 42: 1008-17.

9 Thompson HS. Pupillary signs in the diagnosis of optic nerve disease. Trans Ophthalmol Soc UK 1976; 96: 377-81.

10 Loewenfeld I. In: Whitcomb MA, ed. Recent Developments in Vision Research. Washington: National Research Council Publication, 1966: no. 1272: 17-105. 